EPJ Web of Conferences 31, 00013 (2012)

DOI: $10.1051 /$ epjconf/20123100013

(C) Owned by the authors, published by EDP Sciences - SIF, 2012

\title{
Isovector fluctuations and symmetry energy in fragmentation dynamics
}

\author{
M. Colonna
}

INFN-LNS, Laboratori Nazionali del Sud, 95123 Catania, Italy

\begin{abstract}
Within a dynamical description of nuclear fragmentation, based on the spinodal decomposition scenario, we explore the relation between isovector density fluctuations and nuclear symmetry energy. We show that, inside the fragmenting system, isovector fluctuations follow the evolution of the local density and approach an equilibrium value connected to the local symmetry energy. Higher density domains are characterized by smaller average asymmetry and narrower isotopic distributions. This analysis indicates that fragment isotopic properties and isoscaling can probe the symmetry energy in the density regime corresponding to fragment formation.
\end{abstract}

\section{Introduction}

Under suitable conditions of density and temperature, the nuclear Equation of State (EoS) foresees the possibility of phase transitions from the liquid to the vapour phases, a scenario often evoked to explain the multifragmentation phenomenon [1-3]. In two-component systems, such as asymmetric nuclear matter, where proton and neutron numbers are different, new features appear and a crucial role is played by the isovector part of the interaction and the corresponding term in the nuclear EoS, the so-called symmetry energy [4]. Hence, apart from the its fundamental interest, the analysis of phase transitions in two-component nuclear systems allows one to explore the low-density behavior of the symmetry energy, on which many investigations are concentrated nowadays [5-8].

This is an Open Access article distributed under the terms of the Creative Commons Attribution License 2.0, which permits unrestricted use, distribution, and reproduction in any medium, provided the original work is properly cited. 
A connection between the characteristics of clusters emerging from nuclear fragmentation and the symmetry energy has been proposed, in the framework of macroscopic statistical models $[9,10]$. Here we explore this issue within a dynamical description of the fragmentation process, corresponding to the occurrence of low-density (spinodal) instabilities. We investigate the coupling between the development of isovector density fluctuations, to which isotopic properties are connected, and the growth of the isoscalar unstable modes, leading to the formation of nuclear drops (fragments). This allows one to evaluate isovector fluctuations as a function of the local density inside the fragmenting system, to probe their relation to the symmetry energy and its density dependence.

\section{$2 \quad$ SMF model and isovector fluctuations}

Theoretically the evolution of complex systems can be described by a onebody transport equation with a fluctuating term, that incorporates the effects of the unknown many-body correlations, the so-called BoltzmannLangevin equation (BLE). Here we follow the approximate treatment to the BLE presented in Ref. [12], the Stochastic Mean Field (SMF) model, that consists in the implementation of stochastic spatial density fluctuations. Hence we consider the following equation for the time evolution of the semiclassical one-body distribution function $f(\boldsymbol{r}, \boldsymbol{p}, t)$ :

$$
\frac{\partial f}{\partial t}+\boldsymbol{v} \cdot \frac{\partial f}{\partial \boldsymbol{r}}-\frac{\partial U}{\partial \boldsymbol{r}} \cdot \frac{\partial f}{\partial \boldsymbol{p}}=\bar{I}_{\text {coll }}[f]+\frac{\partial U_{\text {ext }}}{\partial \boldsymbol{r}} \cdot \frac{\partial f}{\partial \boldsymbol{p}},
$$

where $U[\rho]$ is the self-consistent mean-field potential, $\bar{I}_{\text {coll }}[f]$ is the average collision integral and $U_{\text {ext }}(\boldsymbol{r})$ represents the external, stochastic field. The coordinates of isospin are not shown for brevity.

Within such a framework, the effective potential $U$ is derived from energy density functionals that, in asymmetric matter, contain a term proportional to $I^{2}$ (with $I \equiv\left(\rho_{n}-\rho_{p}\right) / \rho$ and $\rho, \rho_{n}, \rho_{p}$ denoting total, neutron and proton densities, respectively), the so-called symmetry energy, $E_{\text {sym }}(\rho, I) / A \equiv C_{\text {sym }}(\rho) I^{2}$. The symmetry energy coefficient $C_{\text {sym }}(\rho)$ can be expressed as the sum of a potential term $C_{s y m}^{p o t}(\rho)$, and a kinetic term that, at zero temperature, reduces to $\epsilon_{F}(\rho) / 3$, where $\epsilon_{F}$ denotes the Fermi energy.

In order to investigate fluctuations of the one-body distribution function $f$ and their dependence on system conditions, such as density and temperature, let us consider the behavior of nuclear matter prepared with a 
uniform density distribution $\rho_{0}$ and with a Fermi-Dirac momentum distribution $f_{0}(\epsilon)$ corresponding to a specified temperature $T$. The system is confined within a volume $\Omega$, with periodic boundary conditions imposed. For two-component matter one can identify two types of independent modes of the phase-space density: isoscalar-like modes, where neutrons and protons oscillate in phase, and isovector-like modes, with neutrons and protons oscillating out-of phase [4]. In particular, in the case of symmetric nuclear matter, the two types of modes correspond to oscillations of the total density $f^{s}=f_{n}+f_{p}$ (isoscalar modes) and of the isovector density $f^{v}=f_{n}-f_{p}$ (isovector modes). Let us denote by $f_{\mathbf{k}}^{q}(\boldsymbol{p}, t)(q=s, v)$ the Fourier transform of the difference $\delta f^{q}=f^{q}-f_{0}^{q}$, where $f_{0}^{q}$ is the system initial density.

For stable modes, for which equilibrium is eventually reached, the variance associated with the density fluctuation $\rho_{\mathbf{k}}^{q}$ can be evaluated exploiting the fluctuation-dissipation theorem [13]:

$$
\sigma_{k}^{q}=<\left(\rho_{\mathbf{k}}\right)^{2}>=\frac{T}{F^{q}(k)},
$$

where $F^{q}(k)=\left(\frac{\partial U_{k}^{q}}{\partial \rho^{q}}+1 / N\right)$, with $N=-\frac{4}{h^{3}} \int d \boldsymbol{p} \frac{\partial f_{0}}{\partial \epsilon}$. We notice that $F^{q}$ is nothing but the second derivative of the system free energy density with respect to the density $\rho^{q}$. Considering the inverse Fourier transform of $\rho_{k}^{q}$ we obtain, for the spacial density correlations, in a cell of volume $\Delta V$ :

$$
\sigma_{\rho^{q}}(\Delta V) \equiv<\delta \rho^{q}(\boldsymbol{r}) \delta \rho^{q}(\boldsymbol{r})>=\frac{1}{(2 \pi)^{3}} \sum_{\mathbf{k}} \sigma_{k}^{q} d \mathbf{k}=\frac{1}{\Delta V}<1 / F^{q}(k)>_{\mathbf{k}},
$$

where the average extends to all $\mathbf{k}$ modes.

It should be noticed that, for the physical scenario we are interested in , i.e. unstable nuclear matter, isoscalar density fluctuations are amplified, while isovector modes are stable and the associated fluctuations are expected to keep the value given by Eq.(3). Focusing on the isovector modes, the potential $U_{k}^{v}$ represents the Fourier transform of the difference between neutron and proton potentials, $U_{\text {sym }}(\rho(\boldsymbol{r}))=\int d \boldsymbol{r}^{\prime} 2 C_{\text {sym }}^{\text {pot }}\left(\rho\left(\boldsymbol{r}^{\prime}\right)\right) I \cdot g_{\sigma}\left(\left|\boldsymbol{r}-\boldsymbol{r}^{\prime}\right|\right)$, where the smearing function $g_{\sigma}$ is introduced to account for the finite range of the nuclear interaction. Then one gets $F^{v}(k)=2 F_{\text {sym }}(k) / \rho_{0}$, with $F_{\text {sym }}(k)=C_{\text {sym }}^{\text {pot }} g_{\sigma}(k)+\rho_{0} /(2 N)$. We note that $F_{\text {sym }}(k=0)$ simply coincides with the (volume) symmetry free energy, that at zero temperature reduces to the symmetry energy $C_{s y m}$, because $1 / N(T=0)=\left(2 \epsilon_{F}\right) /\left(3 \rho_{0}\right)$. Then we can write:

$$
<1 / F^{v}(k)>_{\mathbf{k}}=\frac{\rho_{0}}{2}<1 / F_{\text {sym }}(k)>_{\mathbf{k}} \equiv \frac{\rho_{0}}{2 F_{\text {eff }}^{v}} .
$$


Hence we find that fluctuations of the isovector density are connected to an "effective" symmetry free energy $F_{\text {eff }}^{v}$ that, owing to the $k$ dependence of the symmetry potential, is smaller than the volume free energy $F_{\text {sym }}(k=0)$. For instance, adopting $\sigma=0.9 \mathrm{fm}$, we find $F_{\text {eff }}^{v}=0.7 C_{s y m}$ for nuclear matter at saturation density and zero temperature.

In asymmetric matter, the findings discussed above still hold for isoscalar-like and isovector-like oscillations.

\section{$3 \quad$ Results}

Let us go back to the full non-linear equations (1), that are solved numerically with the test particle method. We have performed SMF calculations for nuclear matter prepared at fixed temperature $(\mathrm{T}=3 \mathrm{MeV})$ and in several density conditions. We adopt a soft EoS, with compressibility modulus $K=200 \mathrm{MeV}$ and, for the density $(\rho)$ dependence of the symmetry energy we consider two representative parameterizations: one with a linearly increasing behaviour of $C_{s y m}^{\text {pot }}(\rho)$ with density (asystiff) and one with a kind of saturation above normal density (asysoft) (see Ref. $[4,14]$ for more detail). The corresponding zero-temperature coefficient $C_{\text {sym }}(\rho)$ is plotted in the inset of Fig.1 (full thick lines).

Let us consider first, for the sake of simplicity, the case of symmetric matter $(I=0)$. We first concentrate on isovector fluctuations for matter at equilibrium. To do so, we switch-off in the calculations the isoscalar part of the nuclear potential, to avoid the development of instabilities in the case of low density initial conditions, and we calculate the isovector fluctuation variance $\sigma_{\rho^{v}}=<\left(\delta \rho_{n}(\boldsymbol{r})-\delta \rho_{p}(\boldsymbol{r})\right)^{2}>$, where the average is performed over cells of volume $\Delta V=1 \mathrm{fm}^{3}$. Then, the effective symmetry free energy can be extracted numerically exploiting Eqs. $(3,4)$. This quantity, $F_{\text {eff,num }}^{v}$, is displayed in Fig.1 (left panel, inset) as a function of the matter density, for the two parameterizations of the symmetry energy introduced above (dot-dashed thin lines, black for asystiff and red for asysoft). It should be noticed that at the small temperature $(T=3 \mathrm{MeV})$ considered in the calculation, the symmetry energy $C_{\text {sym }}$ (thick lines) practically coincided with the symmetry free energy $F_{\text {sym }}(k=0)$. The numerical results follow the analytical calculation discussed above: Owing to the $k$ dependence of the symmetry potential, the extracted $F_{e f f, n u m}^{v}$ is lower than the symmetry energy, being reduced by about $30 \%$ at normal density, but exhibits a similar density dependence, connected to the asy-stiffness of the effective interaction employed in the simulations. A different behavior is seen for densities below 

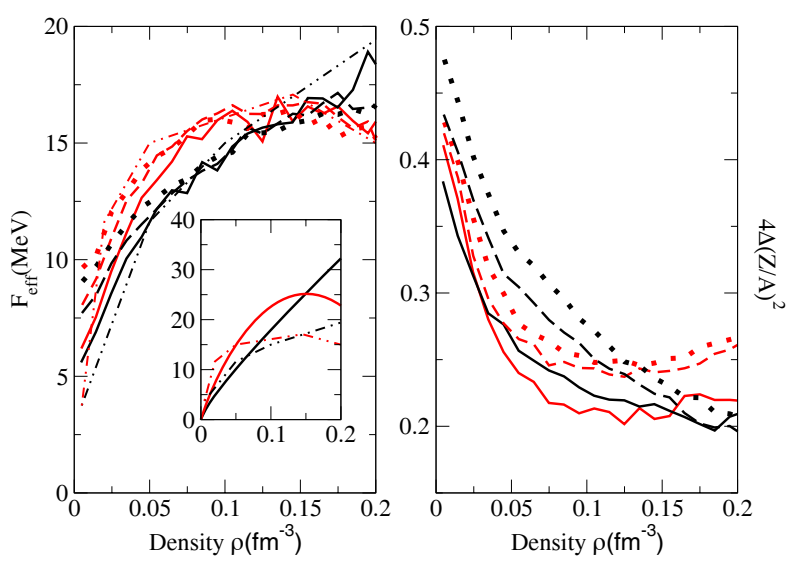

Figure 1: (Color online) Left panel: The effective free symmetry energy, as extracted from SMF simulations, as a function of the local density, for systems with temperature $\mathrm{T}=3 \mathrm{MeV}$ and density $\rho_{1}$ (full lines), $2 \rho_{1}$ (dashed lines), $3 \rho_{1}$ (dotted lines). Dot-dashed lines (also shown in the inset) represent the results obtained for stable nuclear matter at the same temperature (see text). In the inset the density dependence of the symmetry energy coefficient $C_{\text {sym }}$ is displayed, for comparison (full lines). Right panel: The quantity $4 \Delta(\overline{Z / A})^{2}$ ) (see text) is plotted as a function of the local density. Notations are the same as in the left panel. Black: asystiff EoS. Red: asysoft EoS.

$0.05 \mathrm{fm}^{-3}$. Here the calculations could be altered by numerical inaccuracies related to the phase space mapping (that would require a very huge number of test particles), that may induce deviations of the distribution function from the Fermi-Dirac statistics.

The evaluation of the isovector fluctuations of nuclear matter frozen at fixed density can be used as a benchmark for the general and more interesting case where the system may evolve. Solving numerically the full non linear equations (1), one expects a coupling between isoscalar and isovector modes. Then it is interesting to investigate the behavior of isovector fluctuations in presence of instabilities leading to the amplification of isoscalar density fluctuations. Calculations have been performed for three initial density values inside the spinodal region: $\rho_{0}=\rho_{1}=0.0245 \mathrm{fm}^{-3}, \rho_{0}=2 \rho_{1}$ and $\rho_{0}=3 \rho_{1}$. Moreover, for each case, we have considered symmetric matter (system (1), $\left.I_{1}=0\right)$ and asymmetric matter (system (2), $I_{2}=0.142$ ).

Now the system may develop density fluctuations, so locally the density gets larger (density bumps, leading to fragments) or smaller (vapour) than the initial value. Then isovector fluctuations can be investigated as a function of the local density, i.e. looking at the behavior of the isovector density 
$\rho^{v}$ in cells having the same total density $\rho$. This analysis is performed at the time $t=200 \mathrm{fm} / \mathrm{c}$, when isoscalar density fluctuations saturate. Results for $F_{\text {eff,num }}^{v}(\rho)$ are displayed in Fig.1 (left panel), as a function of the local density, in the case of symmetric matter, for the three initial density values considered in the calculations, and the two asy-EoS.

Quite interestingly, one observes that isovector fluctuations follow the local value of the symmetry energy independently of the initial conditions of the system. Indeed the three curves associated with the different initial densities (full, dashed and dotted lines for $\rho_{1}, \rho_{2}$ and $\rho_{3}$, respectively) are rather close to each other and they are also close, for each given density, to the equilibrium results discussed above (thin dot-dashed lines, also shown in the inset). These results indicate that, as soon as density fluctuations start to develop, one observes a quick rearrangement of isovector fluctuations, that approach the equilibrium value corresponding to the actual local density.

Calculations have been performed also for the asymmetric system (2), leading to results very close to the ones displayed in Fig.1. In the latter case one can also discuss the isospin distillation mechanism, that induces a deviation of the local asymmetry from the system initial value. In particular, we consider the quantity $\Delta(\overline{Z / A})^{2}=(\overline{Z / A})_{1}^{2}-\left((\overline{Z / A})_{2}^{2}\right.$, where $(\overline{Z / A})_{1}=1 / 2$ refers to the symmetric system and $(\overline{Z / A})_{2}=\left(1-\bar{I}_{2}(\rho)\right) / 2$ is related to the average asymmetry value of cells having the same local density $\rho$, for system (2). This quantity is displayed in Fig.1 (right) as a function of $\rho$.

The different curves correspond to the two EoS (red lines for soft, black lines for stiff) and the three initial densities considered. As a general trend, we observe the well known behavior of asymmetric systems: the low-density regions become more neutron rich, while high density regions are more symmetric, just in connection with the density dependence of the symmetry energy coefficient $C_{\text {sym }}(\rho)$. Here what is interesting to notice is that the distillation mechanism goes together with the density-dependent behavior of the isovector variances described just above: large density domains are associated with smaller asymmetry and smaller fluctuation width, while low density regions are on average more asymmetric, but also more fluctuating.

\section{Conclusions}

To conclude, we have undertaken a dynamical study of the disassembly of two-component unstable systems, focusing on the coupling between the development of isoscalar and isovector density fluctuations. We have shown that the amplitude of isovector fluctuations follows the evolution of the local 
density and approaches, within time scales compatible with nuclear reactions at Fermi energies, the corresponding local equilibrium value, that is linked to the density-dependent symmetry free energy (see Eqs. $(3,4)$ ). The isospin distillation mechanism, that reduces the asymmetry of the high density domains from which fragments emerge, is accompanied by the observation of smaller isotopic fluctuations in these regions, following the density dependence of the symmetry energy. These results are relevant to experimental isoscaling analyses aiming at extracting information on the symmetry energy, a topic of strong current interest in the nuclear physics community.

\section{References}

[1] D.R. Bowman, G.F. Peaslee, R.T. DeSouza, et al. Phys. Rev. Lett. 67, 1527 (1991)

[2] G. Bertsch and P.J. Siemens, Phys. Lett. B126, 9 (1983)

[3] B. Borderie, M.F. Rivet, Progr. in Part. and Nucl. Phys. 61 (Book Series), 551 (2008)

[4] V. Baran, M. Colonna, V. Greco, M. Di Toro, Physics Reports 410, 335 (2005)

[5] S.R.Souza, M.B.Tsang, arXiv:1112.3631

[6] D.V. Shetty et al., Phys. Rev. C76, 024606 (2007)

[7] G.Lehaut, F.Gulminelli, O.Lopez, Phys. Rev. Lett 102, 142503 (2009)

[8] E.Galichet et al., Phys.Rev. C79, 064615 (2009)

[9] A.Botvina et al., Phys. Rev. C65, 044610 (2002)

[10] M.B.Tsang et al., Phys. Rev. Lett. 86, 5023 (2001)

[11] A.Guarnera, M.Colonna, Ph.Chomaz, Phys. Lett. B373, 297 (1996)

[12] M. Colonna et al, Nucl. Phys. A642, 449 (1998)

[13] L.D. Landau, E.M. Lifshitz, Statistical Physics Part 1. Vol. 5 (3rd ed.), Butterworth-Heinemann, ISBN 978-0- 750-63372-7 (1980)

[14] V. Baran et al., Nucl. Phys. A703, 603 (2002) 